\title{
La communication au service de la gestion des conflits valeurs : « social vs économie? »
}

\section{Violaine Appel}

\section{(2) OpenEdition}

12 Journals

Édition électronique

URL : http://journals.openedition.org/communicationorganisation/2902

DOI : 10.4000/communicationorganisation.2902

ISSN : $1775-3546$

Éditeur

Presses universitaires de Bordeaux

Édition imprimée

Date de publication : 1 mai 2004

ISSN : 1168-5549

Référence électronique

Violaine Appel, «La communication au service de la gestion des conflits valeurs : " social vs

économie ? » », Communication et organisation [En ligne], 24 | 2004, mis en ligne le 27 mars 2012,

consulté le 30 avril 2019. URL : http://journals.openedition.org/communicationorganisation/2902 ;

DOI : 10.4000/communicationorganisation.2902

Ce document a été généré automatiquement le 30 avril 2019.

(c) Presses universitaires de Bordeaux 


\title{
La communication au service de la gestion des conflits valeurs : " social vs économie?»
}

\author{
Violaine Appel
}

1 Ces dernières années, le positionnement des organisations par rapport à la société se caractérise par la prise en compte croissante de problèmes dépassant le seul cadre économique: par exemple, de nombreuses entreprises semblent se soucier de la protection de l'environnement.

2 Or, dans leur vocation à se développer, les organisations cherchent, d'une part, à sensibiliser leurs publics aux objectifs et enjeux qui sont les leurs et, d'autre part, à ajuster les comportements de chacun à un fonctionnement collectif satisfaisant pour tous. De plus en plus, ce souci de la nécessaire adaptation de l'organisation à la complexité croissante de son environnement conduit les structures à ne plus pouvoir envisager leur survie sans recourir à une médiatisation de leur projet.

3 Cette prise de parole conduit notamment les entreprises de l'économie sociale, celles qui sont au service de la société et dont l'objet est l'intérêt collectif de tous les membres et non le profit d'actionnaires, à gérer des situations qui peuvent paraître paradoxales de prime abord. A partir de l'étude du cas de la MAlF, nous tenterons de dégager quelques pistes pour une réflexion plus globale sur les enjeux d'une logique communicationnelle résidant dans une certaine dualité des objectifs, ou encore d'une stratégie de communication au service de la gestion des conflits de valeurs. 


\section{Logiques économique, identitaire et médiatique : un triptyque complexe}

4 Aujourd'hui, les organisations évoluent dans un environnement incertain, marqué par des évolutions rapides, une concurrence internationale croissante, une déréglementation des marchés publics, un envahissement des nouvelles technologies, etc.

Ces nouvelles contraintes, auxquelles l'entreprise ne peut se soustraire, pèsent notamment sur son organisation, sa stratégie de développement et son discours. Elles lui imposent, entre autres, un engagement accru dans des activités qui dépassent son seul rôle économique. La crédibilité et la légitimité de l'entreprise paraissent aussi vouloir se jouer dans sa capacité à se définir socialement, à mettre en évidence son engagement sociétal.

6 Plusieurs auteurs de disciplines variées, de la sociologie aux sciences de gestion, signalent une profonde mutation de l'entreprise depuis quelques décennies: par exemple. Boltanski et Chiapello ${ }^{1}$ pensent que le capitalisme est habité d'un «nouvel esprit » et Crozier $^{2}$ voit le management soumis à une nouvelle logique. Cette mutation s'accompagne de «modes» communicationnel(le)s: entreprise citoyenne, développement durable, notamment. Comme l'explique Nicole d'Almeida «l'entreprise ne rendait traditionnellement compte qu'au cercle restreint du conseil d'administration et à ses actionnaires. La nouveauté des dernières décennies réside dans l'élargissement du champ des paroles émises par l'acteur économique, dans l'émergence de récits qui portent tour à tour sur les produits et les marques (mis en scène par la publicité), sur les hommes de l'entreprise (la communication interne) et enfin sur l'institution elle-même (communication institutionnelle). Ce faisant, les entreprises passent d'une politique d'information à une politique de communication dominée par la dimension phatique, par la création et la relance perpétuelle du contact $»^{3}$. L'entreprise apparaît de plus en plus en prise avec la société.

7 Toutefois, si comme l'évoque Etienne Lissilour ${ }^{4}$ plusieurs approches s'accordent à reconnaître l'influence de la société sur l'entreprise - que ce soit « l'effet sociétal » mis en évidence par des sociologues et économistes du Laboratoire d'Economie et de Sociologie du Travail d'Aix-en-Provence ${ }^{5}$ ou bien l'idée de « culture nationale » évoquée par Philippe d'iribarne $^{6}-$ il n'en reste pas moins que l'on peut aussi se demander si l'entreprise peut rétroagir sur la société. Car. comme le spécifie Etienne Lissillour. "les développements actuels insistent sur l'idée d'une entreprise de plus en plus présente en société et sur le passage d'une entreprise-organisation vers une entreprise-institution: une institution qui vise à instaurer un mode de régulation et, dans ce but. à maintenir et à faire perdurer des valeurs et des modèles de comportements $»^{7}$.

8 Les choix stratégiques effectués et permettant de déterminer des modalités et une trajectoire de développement de l'organisation, doivent donc désormais prendre en compte à la fois une logique de rentabilité immédiate, liée à l'évolution de son environnement, et une logique identitaire relevant d'un objectif de pérennité de l'entreprise, liée à sa liberté de choix d'être ce qu'elle veut être.

9 Ces choix deviennent alors un projet et un langage partagés par tous ceux qui ont un impact sur la stratégie. Et. c'est la mise en système médiatique (tant interne qu'externe) 
de ces choix, traduits en discours, qui permet aux membres de l'organisation de s'y identifier et aux personnes extérieures de la reconnaître.

C'est donc au cœur de la société et donc au cœur d'un nœud complexe d'interférences économiques, identitaires et médiatiques, que se situe le champ d'exercice de l'activité des organisations.

\section{Logique d'appartenance et logique médiatique au service d'une logique de re-connaissance}

11 L'hypothèse qui sous-tend cette recherche est que les contraintes évoquées précédemment imposent aux entreprises de l'économie sociale de faire exister leur propre éthique au sein du système libéral, de faire coexister le monde des valeurs qu'elles défendent avec celui du « tout-économique ».

En d'autres termes, il s'agit ici de comprendre pourquoi et comment une stratégie de communication, résultat d'une volonté politique liée à des impératifs de développement, devient un enjeu éthique, voire philosophique, autour duquel s'organisent les logiques des différents acteurs en présence.

Pour la traiter, nous avons choisi de partir de l'analyse d'un cas bien spécifique: la stratégie de communication de la MAIL entre septembre 2000 et septembre 2002. L'enjeu pour cette mutuelle, sans actionnaire mais avec des sociétaires, est de se développer sans se trahir à travers une campagne engagée. La signification symbolique des services proposés et des valeurs qui y sont rattachées marque à la fois son engagement professionnel et militant (ancré dans une éthique profondément marquée par des valeurs humanistes) et cherche, en même temps, à la démarquer des conceptions économiques et financières : la rentabilité est nécessaire mais non plus suffisante. Plus précisément, dans le cadre de l'économie sociale, l'objectif de rentabilité est inféodé à l'objectif de satisfaction sociale.

Ici, la double influence société-entreprise et entreprise-société existe indubitablement et contraint la MAIL à gérer en permanence cette dichotomie entre engagement moral et système libéral, entre éthique et capital.

15 La campagne de communication mise en place doit en quelque sorte permettre de gérer les changements, liés à des impératifs économiques, dans la continuité des valeurs partagées.

16 Une nouvelle logique d'appartenance: de l'appartenance communautaire à l'appartenance sociétaire en passant par la reconnaissance.

17 Il s'agit en effet de rompre avec le corporatisme originel qui offrait une vision communautaire - certes fondée sur certaines valeurs de solidarité et d'attention mutuelle, mais surtout ancrée dans l'appartenance à un même groupe social (les instituteurs puis les enseignants) - pour s'ouvrir à d'autres publics partageant les mêmes valeurs mutualistes. Il s'agit donc ici de modifier l'attachement à la MAIL en jouant sur la reconnaissance et non plus sur l'appartenance à un même groupe social.

C'est probablement la raison pour laquelle la MAIL adopte un positionnement $\mathrm{d}^{\prime}$ ' assureur militant $»^{8}$. Pour la première fois de son histoire, la stratégie de développement de cette mutuelle a cherché à affirmer avec force un engagement professionnel ancré dans une éthique du respect de la personne. La solidarité, l'attention 
mutuelle, la tolérance, la confiance. la proximité sont alors les conditions sine qua non de sa « philosophie d'entreprise ».

Par ailleurs, cette éthique affiche clairement la primauté de l'homme sur le tout économique. Le fait de posséder des sentiments, des émotions, d'avoir des idées, des convictions et de défendre des engagements, prédomine sur l'idée de matricule, d'adhérent, d'« anonyme derrière les chiffres ».

20 Mais qu'on ne s'y trompe pas. il ne s'agit la que d'un transfert car au final. ce qui est souhaité, c'est bien l'adhésion à lu MAIF (dans les deux sens du terme: adhérents adhérant aux valeurs).

21 A travers le slogan institutionnel: " assureur qui vous ressemble et vous rassemble $»^{9}$ la MAIF joue sur une double proximité : psychologique (nous partageons les mêmes valeurs) mais aussi physique (nous appartenons à la même mutuelle). La MAIF se veut fédératrice ( "rassembler») d'un ensemble de sociétaires, fondé sur une sorte de melting-pot socioprofessionnel (s'ouvrir à d'autres professions) et culturel, animé par le respect de l'homme et le refus de l'exucerbation du capitalisme. Il s'agit de rassembler ceux et uniquement ceux qui partagent les mêmes valeurs ( ressembler »).

Cette logique de reconnaissance, visant à développer la notoriété de la mutuelle en améliorant son image de marque auprès du grand public, n'en constitue pas moins un enjeu «interne». La MAIL a pris soin, avant de commencer sa campagne de communication médiatique en direction du grand public, d'en informer ses sociétaires. Ce métadiscours, s'il avait bien sûr pour but d'informer, comportait d'autres objectifs stratégiques. En effet, si la plupart des sociétaires étaient susceptibles d'être confortés dans leur choix et leurs convictions, le risque était de voir partir ceux qui pouvaient se sentir en "porte-à-faux» avec cette prise de parole publique. Car il ne s'agit pas seulement d'être en accord avec la philosophie développée, il faut aussi s'engager, au quotidien, à être le reflet des valeurs auxquelles on adhère (" redonnons du sens à nos comportements ", " pensez davantage aux autres $\left.{ }^{10}\right)$.

23 Tout comme pour le public externe, on passe alors d'une stratégie de reconnaissance à une stratégie d'appartenance. "avec ce sentiment de fierté qui s'en trouve ravivé », chez les sociétaires, «ceux-ci constituant le meilleur réseau de prescripteurs de la marque MAIF $»^{11}$.

24 D'une certaine façon la révolution est complète: d'une structure corporatiste qui communiquait uniquement par le biais du bouche-à-oreille et du « prosélytisme actif des sociétaires $»^{12}$, on est passé à une structure militante qui compte sur son réseau de sociétaires pour valoriser son image. Il n'y a guère que les mots qui changent. Pour passer de l'une à l'autre, la MAIL a orchestré une campagne de communication qui a modifié la logique d'appartenance en jouant sur une autre forme de reconnaissance : faire passer du désir de connaissance ou de re-connaissance au désir d'appartenance.

\section{Une nouvelle logique médiatique : la prise de parole collective publique}

A l'externe, la MAIF souhaite mettre en avant son image d'assureur militant en cultivant la différence. Il s'agit de maintenir et de protéger sa singularité et d'apparaître comme un assureur différent des autres. Cette différenciation s'exprime certes à travers des valeurs 
mais également la structuration et le ton de la campagne : impertinence du ton et sérieux des sujets abordés.

La stratégie du plan média est axée sur une logique de diffusion du message qui réside dans la dualité des objectifs : fédérer autour de valeurs universelles pour finalement vendre une gamme de services ou de prestations. On passe ainsi progressivement d'une campagne de communication institutionnelle à une campagne de communication produit, car pour la MAIF, il s'agit d'ancrer une valeur dans des engagements concrets, crédibilité oblige!

Les premières insertions presse situent la campagne dans sa dimension idéologique (politique) «nos valeurs les plus précieuses ne sont pas cotées en bourse ${ }^{13}$ et annonce l'ouverture à un plus large public, en ayant pris soin en même temps de "brandir » sa principale valeur, « je ne dirai plus que les profs sont des privilégiés » ${ }^{14}$.

Progressivement les autres insertions presse ou spots télévisuels sont là pour introduire les valeurs chères à la MAIL. Et, tout en recherchant une identification de sociétaires ou futurs sociétaires, la MAIF adopte un ton en décalage avec le discours tenu par le monde des assurances, pour mieux encore s'en différencier.

Les derniers messages, presse ou radio, mettent davantage l'accent sur l'offre en détaillant les contrats, tout en continuant à afficher la valeur maîtresse de la MAIF : le respect de l'homme et sa primauté sur le tout économique.

Ce qui revêt de l'importance, tant pour cette mutuelle qui cherche l'efficacité, que pour l'adhérent qui y trouve une satisfaction de ses besoins, c'est la signification symbolique des prestations proposées et des valeurs qui y sont rattachées en une image cohérente.

31 Ces trois temps «politiques » vont de pair avec les trois phases «médiatiques » de cette campagne. La première phase appelée phase de lancement, utilise exclusivement la presse quotidienne nationale et régionale, médias par lesquels l'exposition aux annonces résulte le plus souvent d'une démarche volontaire. L'affirmation des valeurs « humanistes » de la MAIF va donc toucher ceux qui vont « accepter » d'y être confrontés. La seconde phase, appelée phase d'insistance, utilise la télévision, média plus puissant, touchant un public plus large et impliquant une exposition plus ou moins passive au message. La troisième phase, appelée phase de persistance, utilise la presse magazine et la radio, médias pour lesquels «exposition" n'est pas forcément synonyme de «consommation» (programme TV. salle d'attente, voiture, etc.). La MAIF a ensuite effectué plusieurs " piqûres de rappel » avec une reproduction des phases 2 et 3.

Cette campagne s'inscrit d'ailleurs dans le système éthique précédemment évoqué. En effet, la proclamation de valeurs et non de tarifs permet à la MAIL de jouer sur l'affect. de faire appel aux sentiments, d'affirmer clairement son refus d'une société dominée par l'argent et donc de refuser la logique promotionnelle de ses concurrents. La valorisation de-valeurs «humanistes» et non lucratives lui permet de réaffirmer la primauté de l'homme sur le profit. Le choix des supports traduit également un choix qualitatif d'audience, donc d'un public désiré, ainsi que la volonté de s'associer ou non à certaines lignes éditoriales. Par exemple, l'absence de TF1 et la forte présence de Radio France témoignent de la démarche adoptée par la MAIF.

Par ailleurs, ses actions extérieures rendent crédible l'image qu'elle veut donner à ses services: ses différents partenariats avec la fédération française des Sociétés d'Aviron, une grande majorité des associations clientes des banques populaires et avec le groupe Association Générale de Prévoyance Militaire (AGPM) renforcent l'image institutionnelle, 
fondée-sur l'entraide, le soutien désintéressé et l'action et permettent d'asseoir l'authenticité de ses propos.

\section{Une logique de double contrainte contournée ou la gestion des conflits de valeurs} présente comme celle qui est au service de la société, au service des interêts collectifs de ses sociétaires, qui sont définis par ce qu'ils «sont». et non au service du profit d'actionnaires, qui sont définis par ce qu'ils « ont ». Comme le dit André Comte-Sponville ${ }^{15}$ «cela ne dispense pas une entreprise mutualiste d'être rentable, ni une entreprise capitaliste de satisfaire ses clients. Mais la hiérarchie n'est pas la même. Toute compagnie d'assurance, par exemple, est l'ondée sur la mutualisation des risques. C'est donc une forme de solidarité. Chez un assureur capitaliste, la solidarité est mise au service du profit. Chez un assureur mutualiste, à l'inverse, c'est la rentabilité qui doit être mise au service de la solidarité. Et cette dernière relève de la convergence d'intérêts. Chacun ne peut trouver son propre intérêt qu'en défendant aussi celui des autres ".

38 Et si la MAIF a décide de communiquer, c'est pour asseoir sa notoriété, assurer sa pérennité et mettre sa rentabilité au service de ce qui n'est pas à vendre : ses valeurs.

Dans une société qui tend de plus en plus vers l'individualisme, il fait bon pouvoir se sentir épaulé, soutenu : la MAIF propose la solidarité. Dans une société où l'on dit que chaque jour la violence gagne du terrain, il fait bon se sentir respecté : elle s'engage sur le respect de la personne. Dans une société où les mœurs évoluent, où les divergences ont parfois du mal à s'exprimer, il fait bon être accepté : elle défend la tolérance.

40 Ici aussi la révolution est complète: si les valeurs fondatrices de la MAIF ancrent la campagne de communication dans sa dimension politique, elles sont également l'aboutissement d'une promesse sociétale.

41 En définitive ce que nous montre ici la MAIF c'est qu'il n'y a pas nécessairement une opposition entre un humanisme intégralement bon au service de la vérité et une communication-manipulation mauvaise au service d'un système libéral. L'on peut en effet concevoir que si ces deux aspects semblent parfois contradictoires, ils peuvent s'avérer 
aussi complémentaires, à condition d'éviter le décalage entre image et réalité, à condition que les actes soient en conformité avec les discours, à condition d'ancrer des impératifs de développement dans une éthique du respect de l'homme. ${ }^{16}$

A ce titre la MAIF propose un schéma bien spécifique dans le sens où elle projette un modèle social en utilisant la voie médiatique. Là où certaines entreprises utilisent des valeurs dites «d'entreprise citoyenne» comme message ou simplement comme cadre dans lequel s'inscrit leur politique, la MAIF refuse cette communication «décorum » et propose son éthique comme projet de société.

Il ne s'agit pas simplement de considérer la citoyenneté d'entreprise ${ }^{17}$ comme émanant uniquement de la sphère managériale mais comme une conception partagée à la fois par la structure et par le citoyen, en tenant compte de sa perception, de son bien-être à plus long terme et non uniquement de la satisfaction immédiate de ses besoins en tant que consommateur.

En projetant un modèle sociétal à travers une campagne de-communication, on se différencie d'une stratégie de communication autour d'une thématique de citoyenneté d'entreprise. Il ne s'agit plus d'un discours tenu de l'un à l'autre via les médias mais d'un projet commun et partagé que l'on médiatise.

Par exemple la MAIF ne se contente pas d'offrir des prestations d'assurance pour la famille en réponse à une nécessité mais, à travers ses contrats, elle propose et dans une certaine mesure remet en cause ou interroge la notion même de famille, en la replaçant au cœur des problématiques de société. « Pour la MAIF, un foyer a toujours été un foyer et ce, quelles que soient les personnes qui y vivent, leur statut marital ou leur orientation sexuelle (...). La MAIF n'a pas attendu la création du PACS pour reconnaître et assurer les couples non mariés $»^{18}$.

Si la MAIF se positionne comme précurseur, c'est que ce choix est exigeant et difficile. En effet, se placer sur ce terrain en terme d'image représente une prise de risque.

Tout d'abord, il s'agit d'un terrain "glissant » car fortement marqué idéologiquement même si la MAIF se défend «d'afficher en aucune façon une politique politicienne, un engagement syndical ni une quelconque autre obédience ${ }^{19}$. Cette "alternative à la pensée unique, au tout économique $»^{20}$ traduit une conception de l'assurance différente, à travers un discours volontairement filtrant. Tout le monde ne peut pas s'y reconnaitre.

Ensuite c'est un terrain où la différence n'existe et ne vaut que parce que l'on est les premiers à investir ce champ.

Enfin, cette démarche est extrêmement exigeante dans la mesure où elle est difficile à respecter dans une stratégie cohérente sur le long terme. Il ne peut s'agir d'un simple discours. Il faut que l'ensemble des actes soit conforme aux engagements pris. La MAIF met ses actes en conformité avec ses discours tant au niveau de la relation avec le sociétaire, au niveau des prestations proposées qu'au niveau de l'éthique appliquée au métier, au regard des récents scandales révélés par l'actualité économique et financière.

50 Si aujourd'hui le mode d'expression citoyenne se déplace du politique vers le «hors politique ", l'engagement citoyen doit trouver d'autres formes d'expression. Il peut donc être nécessaire, pour protéger un marché et/ou se protéger sur un marché, de proposer un système de valeurs qui permette aux citoyens de s'exprimer en dehors de la voie politique. 
51 En proposant une alternative crédible et viable, l'économie à vocation humaine, cette mutuelle, avec tous les organismes de l'économie sociale, contribue à la constitution d'un projet de transformation sociale.

Dès lors, on peut penser qu'un changement profond va affecter les politiques des entreprises et des organisations dans les années à venir : il ne suffira sans doute bientôt plus de s'adapter à son environnement et les valeurs médiatisées sans valeur ajoutée au projet de société n'auront peut être plus longtemps la primauté.

\section{BIBLIOGRAPHIE}

APPEL. V., Le dépassement de l'objectif économique par la «valeur sociale ». Analyse des représentations de l'entreprise citoyenne : le cas de la fondation Générale des Eaux (Vivendi). Communication, Editions Nota Bene. Volume 19, n² 2, hiver 1M99-2000. p. 145-154.

BELOT. R.. Je revendique pour la MAIF une prise de parole publique. MAIF Infos, $\mathrm{n}^{\circ} 119$, septembre 2000. p. 6-7.

BELOT. R., Le respect au quotidien. MAIF Infos, $\mathrm{n}^{\circ} 126$, juin 2002, $\mathrm{p} 1$.

BOLTANSKI. L., CHIAPELLO, E. Le Nouvel Esprit du Capitalisme. Paris : Gallimard, 1999.

COMTE-SPONVILLE. A., Aucune société ne peut se passer de valeurs. MAIF Infos, $\mathrm{n}^{\circ} 127$, septembre 2002. p. 14-15.

CROZIER, M., L'entreprise à l'écoute : apprendre le management post-industriel. Paris : InterEditions. 1989. $217 \mathrm{p}$.

D’ALMEIDA, N., Les promesses de la communication. Paris : PUF, sciences modernités philosophies, 2001. $264 \mathrm{p}$.

D'IRIBARNE, P., La logique de l'Honneur. Gestion des entreprises et traditions nationales. Paris : Seuil. Points Essais. 1993. 279 p.

LISSILLOUR, E., De la théorie des organisations à la sociologie de l'entreprise. Management et organisation des entreprises, juillet-septembre 1998, $n^{\circ} 287$, Cahiers Français. La documentation française, p 22-29.

MAIF Infos. La MAIF de retour dans les médias. MAIF Infos, $\mathrm{n}^{\circ} 123$. septembre 2001. p 5.

MAIF Infos. Votre mutuelle plus connue et mieux connue. MAIF Infos, $\mathrm{n}^{\circ} 129$, mars 2003, p 4.

MAIF Infos, La MAIF entre en communication. MAIF Infos, $\mathrm{n}^{\circ}$ 119. septembre 2000. pp. 9-11 et site Internet www.maif.fr (consulté le 21/11/2000).

MAURICE. M., SELLIER. F., SILVESTRE J-J., Politique d'éducation et organisation industrielle en France et en Allemagne. Paris : PUF, 1982.

TRENCIA, B., Nous nous ancrons dans le quotidien le plus vivant : l'homme. MAIF Infos, $\mathrm{n}^{\circ} 119$, septembre 2000. p 8 . 


\section{NOTES}

1. Boltanski L., Chiapello E., Le Nouvel Esprit du Capitalisme. Paris Gallimard. 1999.

2. Crozier, M.. L'entreprise a l'écoute apprendre le management post-industriel. Paris InterEditions. 1989.

3. D’Almeida. N. Les promesses de la communication. Paris: PUF, sciences modernités philosophies. 2001. p 98.

4. Lissillour. E., De la théorie des organisations a la sociologie de l'entreprise Management et organisation des entreprises, juillet-septembre 1998. $\mathrm{n}^{\circ}$ 287. Cahiers français. La documentation française, pp. 22-29.

5. Maurice. M. Sellier. F., Silvestre. J-.J., Politique d'éducation et organisation industrielle en France et en Allemagne. Paris : PUF. 1982.

6. D'iribarne. P., l.a logique de l'Honneur, Gestion des entreprises et traditions nationales. Paris Seuil. Points Essais. 1993.

7. Lissillour. E Opus cit.

8. Trencia. B., Nous nous ancrons dans le quotidien le plus vivant : l'homme. MAIF Infos. $\mathrm{n}^{\circ} 119$. septembre 2000. p. 8

9. MAIF Infos. La MAIF de retour dans les médias. MAIF Infos. $n^{\circ} 123$, septembre 2001. p. 5.

10. Belot. R., Le respect au quotidien Editorial, MAIF Infos, $n^{\circ}$ 126. juin 2002. p. 1

11. MAIF Infos. Votre mutuelle plus connue el mieux connue MAIF Infos. $n^{\circ} 129$. mars 2003. p. 4.

12. Belot, R., Je revendique pour la MAIF une prise de parole publique MAIF Infos, $\mathrm{n}^{\circ} 119$. septembre 2000. p. 6.

13. MAIF- Infos. La MAIF entre en communication MAIF-Infos, $\mathrm{n}^{\circ} 119$. septembre 2000. pp. 9-11. et site Internet www.maif fr (consulté le 21/11/2000).

14. Idem.

15. Comte-Sponville, A., Aucune société ne peut se passer de valeurs. MAIF Infos. $\mathrm{n}^{\circ} 127$. septembre 2002. pp. 14-15.

16. Un précédent travail portant sur l'analyse des représentations de l'entreprise citoyenne a travers la Fondation Vivendi nous avait permis de montrer comment, a l'époque, celle thématique servait de faire-valoir social a une entreprise dont le seul objectif était une recherche croissante du profit

Appel. V. Le dépassement de l'objectif économique par la "valeur sociale » Analyse des représentations de l'entreprise citoyenne: le cas de la Fondation Générale des Eaux (Vivendi) Communication, Editions Nota Bene. Volume 19. $\mathrm{n}^{\circ}$ 2, hiver 1999-2000. pp. 145-154.

17. Nicole D’Almeida précise que « la notion de citoyenneté d'entreprise ne saurait être réduite a un phénomène de mode, assimilée a une éthique ou comparée à une nouvelle forme de philanthropie (...). Si elle peut être comprise comme la poursuite de l'économie par d'autres moyens, la notion renvoie moins à une économie du don qu'a une économie de la responsabilité ou de la responsabilisation II s'agit pour le secteur marchand de faire un détour oblige par le non-marchand afin de fonctionner, de survivre ou de se développer »

D'Almeida. N., Opus cit.. p. 124-125.

18. Site Internet www.maif.fr. consulte le $21 / 11 / 2000$.

19. Trencia, B. Opus cit.

20. Belot. R. Je revendique pour la MAIF une prise de parole publique. MAIF Infos. $\mathrm{n}^{\circ} 119$. septembre 2000. p. 7 


\section{AUTEUR}

VIOLAINE APPEL

Université Nancy 2 